\title{
The Equivalence of Perpendicular Magnetic Recording and Magnetic Force Microscopy
}

\author{
C. D. WRIGHT ${ }^{1}$, E. W. HILL ${ }^{1}$ and R. VLUTTERS ${ }^{2}$
}

1) The Manchester School of Engineering, University of Manchester, Manchester M13 9PL, UK

2) MESA Research Institute, University of Twente, $7500 \mathrm{AE}$ Enschede, Netherlands

\begin{abstract}
A reciprocal force approach is used to demonstrate the equivalence between the (DC) MFM imaging process and the inductive readout process in magnetic recording. For the particular case of a perpendicularly magnetized medium it is shown that MFM imaging via a rectangular tip is entirely equivalent to readout via a Karlquist ring-type head having a gap width equal to the MFM tip width
\end{abstract}

Key words: Magnetic force microscopy, perpendicular recording

\section{INTRODUCTION}

Magnetic force microscopy (MFM) is a powerful tool for the investigation of magnetic structure in a variety of scientifically and technologically important materials. It has proved particularly useful in the study of written transitions in both longitudinal and perpendicular magnetic recording media [1]. Indeed, several recent works have noted strong similarities, in both time and frequency domains, between the MFM image signals of written transitions and the actual readback voltage obtained in a real recording system $[2,3]$. Such similarities arise simply because the MFM imaging process is a direct equivalent of the readout process in magnetic recording. MFM imaging exhibits all the well-known frequency-dependent loss terms, i.e. gap-loss, spacing-loss and thickness-loss, that are so familiar to those working in the field of magnetic recording. In this paper we demonstrate such an equivalence by using a reciprocity-based approach to describe MFM response. In particular we concentrate on perpendicularly magnetized media and show that in this case MFM imaging with a rectangular or single-pole type head is analogous to inductive readout via a Karlqvist-type ring head.

An important consequence of this work is that it shows that MFM imaging offers no inherent improvement in resolution over conventional readback techniques employed in magnetic recording; any improvements that do arise are due simply to a scaling effects of tip (head) and tip-sample separation (head flying height).

\section{RECIPROCITY THEORY}

The reciprocity approach calculates the force acting on the sample, rather the force acting on the MFM tip. These two forces are, by Newton's Third Law, equal but opposite. The reciprocal force acting in the vertical, $z$, direction on the sample can be written as [4]

$$
F_{z}^{\text {samp }}=-F_{z}^{t i p}=\int_{\text {samp } i} \sum_{i} M_{i}^{\text {samp }}\left(r^{\prime}\right) \frac{\partial H_{i}^{t i p}\left(r+r^{\prime}\right)}{\partial z} d^{3} r^{\prime}
$$

where $i$ represents $x, y$ or $z$ directions, $M_{i}^{\text {samp }}(r)$ is the $x, y$ or $z$ component of the magnetization of a sample volume element and $H_{i}^{t i p}\left(r+r^{\prime}\right)$ is the stray field from the tip at the position of that same sample volume element. Equation (1) applies to a force or DC type MFM and assumes that there are no mutual tip-sample magnetization disturbances. Force-gradient or AC MFM systems are modelled by taking in Eq.(1) the second derivative, with respect to $\mathrm{z}$, of the tip stray field. An important feature of the reciprocal force method is that it reveals explicitly the role of the tip field in the imaging process.

For our current purposes, we consider the MFM system shown in Fig. 1. A (infinitely long) rectangular tip of width $2 T$ and magnetized uniformly in the vertical, $z$, direction is used to image a perpendicular magnetic transition, $M_{\mathrm{z}}^{\text {samp }}(x)$, in a film of thickness $D$ and with a tipsample separation of $d$. We initially assume that the tip field and sample magnetization are invariant in the crosstrack, $y$, direction; such a 2-D treatment also being common in most studies of the readout process in magnetic recording.

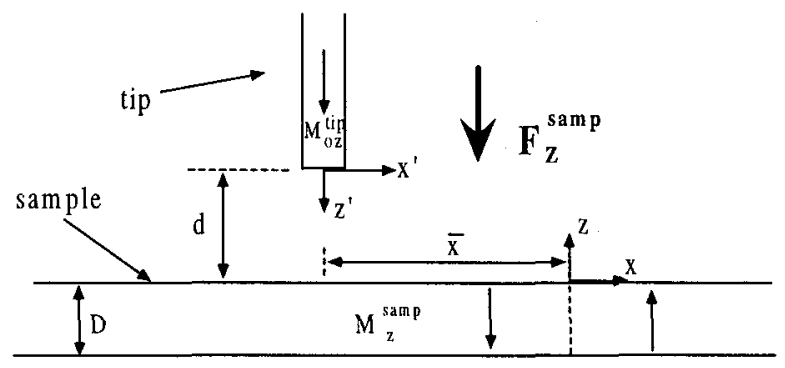

Fig. 1. MFM tip and sample geometry and co-ordinates.

The reciprocal force per unit width (in the $y$ direction) acting on the sample in this case is therefore

$$
F_{z}^{s a m p}(\bar{x})=\int_{-\infty}^{\infty} \int_{d}^{d+D} M_{z}^{\text {samp }}\left(x^{\prime}-\bar{x}\right) \frac{\partial H_{z}^{t i p}\left(x^{\prime}, z^{\prime}\right)}{\partial z^{\prime}} d z^{\prime} d x^{\prime}
$$

where $H_{z}{ }^{\text {tip }}\left(x^{\prime}\right)$ is the $z$ component of field from the tip and tip co-ordinates $\left(x^{\prime}, z^{\prime}\right)$ are related to sample co-ordinates 
$(x, z)$ by the transformations $x=x^{\prime}-\bar{x}, \bar{x}=u\left(t-t_{0}\right)$, where $u$ is the velocity at which the sample moves past the tip and at time $t=t_{o}$ we have $x=x^{\prime}$.

If we assume for the moment that the spacing between the tip and the sample is much greater than the sample thickness, such that the tip field gradients are effectively constant throughout the sample thickness, then (2) becomes

$$
F_{z}^{s a m p}(\bar{x})=D \int_{-\infty}^{\infty} M_{z}^{s a m p}\left(x^{\prime}-\bar{x}\right) \frac{\partial H_{z}^{t i p}\left(x^{\prime}, z^{\prime}=d\right)}{\partial z^{\prime}} d x^{\prime}
$$

that is a correlation of the sample magnetization and the derivative of the tip field at the surface of the sample. As before, for the force-gradient type MFM we would use, in Eqs. (2) and (3), the second derivative of the tip stray field.

The similarity of Eqs. (2) and (3) to the reciprocity integrals used to describe the inductive readout process will not go un-noticed by those familiar with magnetic recording. For example, the magnetic recording equivalent to $\mathrm{Eq}$. (3) describing the readout voltage $e_{\mathrm{z}}$ (per unit turn and per unit track width in the $y$ direction) arising from an identical sample magnetization distribution as above but with the MFM tip replaced by an inductive readout head is

$e_{z}(\bar{x})=-\mu_{0} u D \int_{-\infty}^{\infty} \frac{\partial M_{z}^{s a m p}\left(x^{\prime}-\bar{x}\right)}{\partial \bar{x}} H_{z}^{h e a d}\left(x^{\prime}, d\right) d x^{\prime}$

where $H_{z}^{\text {head }}$ is the head field that would be produced by the readout head if it were energized by unit current [5]. It is clear that Eqs. (3) and (4) are not exactly equivalent, since in the former we differentiate the (tip/head) field with respect to $z$, whereas in the latter it is the magnetization that is differentiated and with respect to the $x$ direction. However, if we move to a frequency domain ( $k$ space) description of Eqs. (3) and (4) we see that they do in fact describe processes with nearly identical transfer functions.

In the spatial frequency ( $k$ space) domain, the MFM reciprocal force is in general given by taking the Fourier transform of Eq. (1). For the simplified system of Eq. (3) we have $[6,7]$

$$
F_{z}^{\text {samp }}(k)=D M_{z}^{\text {samp }}(k)^{*} \frac{\partial}{\partial z^{\prime}} H_{z}^{t i p}\left(k, z^{\prime}=d\right)
$$

where $k$ is the wavenumber (spatial frequency) and * denotes complex conjugation. Now, in $k$ space the field at a distance $z$ below the surface of a two-dimensional field source in free space is simply equal to the field at the surface of that source multiplied by an exponential spacing loss term $\exp (-|k| z),[8(\mathrm{p} 40)]$. Under these circumstances Eq. (5) becomes

$$
F_{z}^{\text {samp }}(k)=-|k| D M_{z}^{\text {samp }}(k)^{*} H_{z}^{t i p}\left(k, z^{\prime}=0\right) \exp (-|k| d)(6)
$$

and we see that the system transfer function is simply $|k| D$ times the product of the transform of the surface field of the tip and the exponential loss factor due to the tip-sample spacing. Likewise, transforming Eq. (4) into $k$ space yields the functionally similar result $e_{z}(k)=-j k \mu_{0} u D M_{z}^{s a m p}(k)^{*} H_{z}^{\text {head }}(k, 0) \exp (-|k| d)$

Thus, subject to our initial assumptions, the process of image formation in the force type MFM and the inductive readout process in magnetic recording have almost identical transfer functions. In magnetic recording circles the exponential loss factor in Eq. (7) is usually called the Wallace spacing loss and leads to a characteristic $54.6(d / \lambda)$ $\mathrm{dB}$ loss where $\lambda$ is wavelength $[8$ ( $\mathrm{p} 40)]$.

For the force-gradient type MFM the situation is only slightly more complicated than as described above in so much as double-differentiation, with respect to $z$; of the tip field yields a transfer function that is $|k|$ times that of the force type MFM.

For cases in which we cannot ignore the variation of the tip stray field gradients throughout the sample thickness, we need to transform Eq. (2) into $k$ space and this yields

$$
F_{z}^{\text {samp }}(k)=M_{z}^{\text {samp }}(k)^{*} H_{z}^{t i p}(k, 0) \int_{d}^{d+D} \frac{\partial}{\partial z^{\prime}} \exp \left(-|k| z^{\prime}\right) d z^{\prime}(8)
$$

for which the solution is straightforward and can be written

$$
\begin{aligned}
F_{z}^{s a m p}(k)=M_{z}^{s a m p}(k)^{*} H_{z}^{i i p}(k, 0) \exp (-|k| d) \\
\cdot(\exp (-|k| D)-l)
\end{aligned}
$$

The system transfer function now contains an additional term due to the finite thickness of the sample. The additional term is usually known as the thickness loss and is often written as $k D\{[1-\exp (-k D)] / k D\}$ where the $k D$ term represents a $6 \mathrm{~dB}$ per octave rise in the overall response and the term in curly brackets represents a loss with respect to this $6 \mathrm{~dB}$ per octave rise, [8 (p 154)]. Again, Eq. (9) is virtually identical to the equivalent magnetic recording case and the force-gradient type MFM has a response that is $|k|$ times that of Eq. (9).

\section{RESULTS AND DISCUSSION}

As an example of the use of the reciprocal force method we calculate the response due to an ideal perpendicular step transition as illustrated in Fig.1. The magnetization is represented as

$$
M_{z}^{\text {samp }}(x)=M_{o z}^{\text {samp }} \operatorname{sgn}(x)
$$

and the simple rectangular 'monopole' tip has a $z$ component of field below the tip (i.e. $z^{\prime}>0$ ) given by, [5],

$$
H_{z}^{t i p}\left(x^{\prime}, z^{\prime}\right)=\frac{M_{o z}^{t i p}}{2 \pi}\left(\tan ^{-I} \frac{T+x^{\prime}}{z^{\prime}}-\tan ^{-1} \frac{T-x^{\prime}}{z^{\prime}}\right)
$$

that is instantly recognisable as equivalent to the $x$ component of head field produced by a Karlqvist-type ring head. 
For the step magnetization then, the reciprocal force is, following from Eqs (2) and (10)

$$
\begin{aligned}
& F_{z}^{s a m p}(\bar{x})=M_{o z}^{s a m p}\left(\int_{-\infty}^{\bar{x}}-\left.H_{z}^{t i p}\left(x^{\prime}, z^{\prime}\right)\right|_{z^{\prime}=d} ^{z^{\prime}=d+D} d x^{\prime}\right. \\
& \left.+\left.\int_{\bar{x}}^{\infty} H_{z}^{\text {tip }}\left(x^{\prime}, z^{\prime}\right)\right|_{z^{\prime}=d} ^{z^{\prime}=d+D} d x^{\prime}\right)
\end{aligned}
$$

and substitution for $H_{z}^{t i p}$ from Eq. (11) results in the analytic solution

$$
\begin{aligned}
F_{z}^{\text {samp }}(\bar{x}) & =\frac{M_{o z}^{\text {samp }} M_{o z}^{t i p}}{2 \pi}\left[\left((T+\bar{x}) \tan ^{-1} \frac{T+\bar{x}}{z^{\prime}}\right.\right. \\
& -(T-\bar{x}) \tan ^{-1} \frac{T-\bar{x}}{z^{\prime}}+\frac{z^{\prime}}{2} \log \left(z^{\prime 2}+(T-\bar{x})^{2}\right) \\
& \left.\left.-\frac{z^{\prime}}{2} \log \left(z^{\prime 2}+(T+\bar{x})^{2}\right)\right)\left.\right|_{z^{\prime}=d} ^{z^{\prime}=d+D}\right]
\end{aligned}
$$

This response is shown in Fig. 2 for a range of medium thicknesses $(0.1,1$ and $10 \mu \mathrm{m})$ and assuming a tip-sample separation of $50 \mathrm{~nm}$ and a tip width (2T) of $20 \mathrm{~nm}$. The results are in fact identical to those obtained via conventional inductive readout of the same transition with a Karlqvist ring-type head. This can be demonstrated via the standard reciprocity integrals used in magnetic recording, whence the voltage (per unit track width) induced in a Karlqvist ring head of gap-width $2 \mathrm{~g}$ is normalized

MFM signal

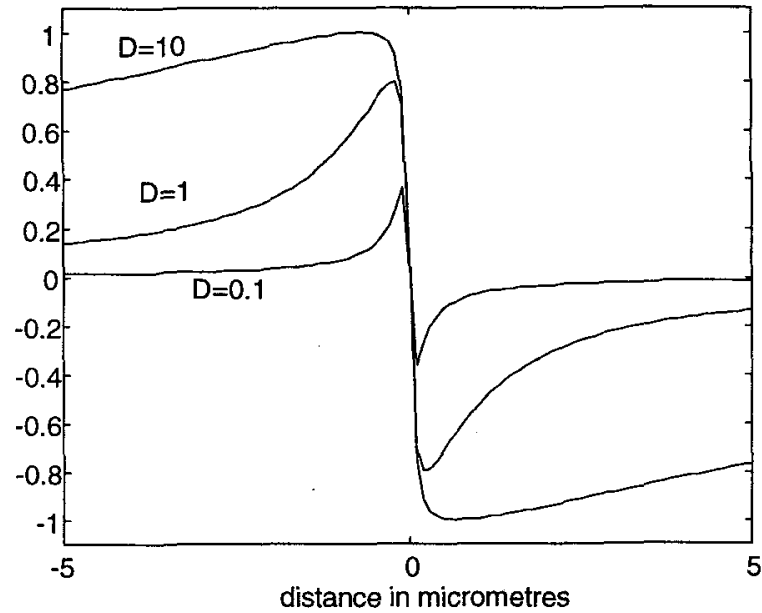

Fig. 2 MFM response to an isolated perpendicular step magnetization in media of thickness $0.1,1$ and $10 \mu \mathrm{m}$.

$$
e_{z}(\bar{x})=-\mu_{0} u \int_{-\infty}^{\infty} \int_{d}^{d+D} \frac{\partial M_{z}^{\text {samp }}\left(x^{\prime}-\bar{x}\right)}{\partial \bar{x}} H_{z}^{\text {head }}\left(x^{\prime}, z^{\prime}\right) d z^{\prime} d x^{\prime}
$$

and $H_{z}^{\text {head }}$ in this case is of the well-known form

$$
H_{z}^{\text {head }}\left(x^{\prime}, z^{\prime}\right)=-\frac{H_{g}}{2 \pi} \log \left(\frac{\left(g+x^{\prime}\right)^{2}+z^{\prime 2}}{\left(g-x^{\prime}\right)^{2}+z^{\prime 2}}\right)
$$

where $\mathrm{H}_{\mathrm{g}}$ is the gap field per unit current in the winding. Thus,

$$
e_{z}(\bar{x})=\mu_{0} u M_{o z}^{\operatorname{samp}} \int_{d}^{d+D} \frac{H_{g}}{2 \pi} \log \left(\frac{\left(g+x^{\prime}\right)^{2}+z^{\prime 2}}{\left(g-x^{\prime}\right)^{2}+z^{\prime 2}}\right) d z^{\prime}(16)
$$

the solution of which is, apart from constant factors, identical to that of Eq (13) [9].

Whilst this direct correspondence between MFM imaging via a rectangular tip and inductive readout via a ring head may at first come as a surprise, their equivalence has in fact already been revealed by Eqs. (5) to (9) where it was shown that in $k$ space the two processes have transfer functions, ignoring constant factors, related by

$$
\frac{F_{z}^{\text {samp }}(k)}{e_{z}(k)}=\frac{|k|}{j k} \frac{H_{z}^{t i p}(k, 0)}{H_{z}^{\text {head }}(k, 0)}=-j \operatorname{sgn}(k) \frac{H_{z}^{\text {tip }}(k, 0)}{H_{z}^{\text {head }}(k, 0)}
$$

Now, it is well-known that the $x$ and $z$ components of a Kalqvist-type field source are themselves a Hilbert transforms, so we can write, [8 (p 43)],

$$
H_{z}^{\text {head }}(k)=-j \operatorname{sgn}(k) H_{x}^{\text {head }}(k)=j \operatorname{sgn}(k) H_{z}^{\text {tip }}(k)
$$

thus

$$
e_{z}(k)=-F_{z}^{s a m p}(k)=F_{z}^{t i p}(k)
$$

and so MFM imaging via a rectangular monopole tip and inductive readout via a ring head have identical transfer functions.

So far we have limited our discussion to a twodimensional system. However, in practice MFM tips have similar dimensions in both $x$ and $y$ directions (i.e. along and across a 'track') and so a 3-D treatment is more appropriate. Indeed, in magnetic recording systems as track widths narrow it is also becoming increasingly important to consider 3-D effects. Extension to 3-D is easily achieved by calculating the fields below the MFM tip using Laplace's equation in free space to represent the magnetic scalar potential, i.e.

$$
\nabla^{2} \phi=0 \text { and } H=-\nabla \phi
$$

Using Fourier transform techniques it is then straightforward to show that, $[10,11]$,

$$
H_{z}^{t i p}\left(k_{x}, k_{y} z^{\prime}\right)=H_{z}^{t i p}\left(k_{x}, k_{y}, 0\right) \exp \left(-\sqrt{k_{x}^{2}+k_{y}^{2}} z^{\prime}\right)
$$

and so the 3-D extension of Eq. (9) becomes

$$
\begin{gathered}
F_{z}^{\text {samp }}\left(k_{x}, k_{y}\right)=M_{z}^{\text {samp }}\left(k_{x}, k_{y}\right)^{*} H_{z}^{t i p}\left(k_{x}, k_{y}, 0\right) \\
\cdot \exp \left(-d \sqrt{k_{x}^{2}+k_{y}^{2}}\right)\left[\exp \left(-D \sqrt{k_{x}^{2}+k_{y}^{2}}\right)-I\right]
\end{gathered}
$$

We have used Eq. (22) to predict the DC-MFM image of perpendicularly magnetized circular 'bit', as might be found for example in magneto-optic storage discs. The results are shown in Fig. 3, again for a range of media 
thicknesses. As an experimental comparison we show in Fig. 4 the MFM image of a pseudo-circular bit thermomagnetically recorded into a $150 \mathrm{~nm}$ thick $\mathrm{Co} / \mathrm{Pt}$ multilayer sample for magneto-optic recording applications. This image was obtained using a Burleigh AFM adapted for MFM use [12].
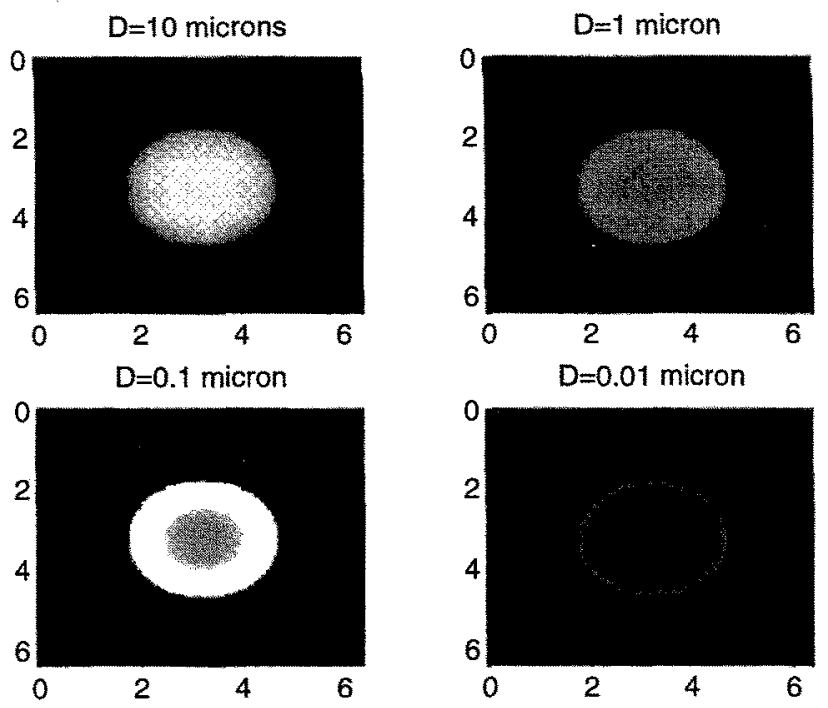

Fig. 3. Predicted DC-MFM images obtained via the reciprocal approach for a perpendicularly magnetized circular 'bit' in media of different thicknesses imaged by a rectangular tip of cross-section $100 \times 100 \mathrm{~nm}$; scan area is $6 \times 6 \mu \mathrm{m}$, tip-sample separation is $50 \mathrm{~nm}$.

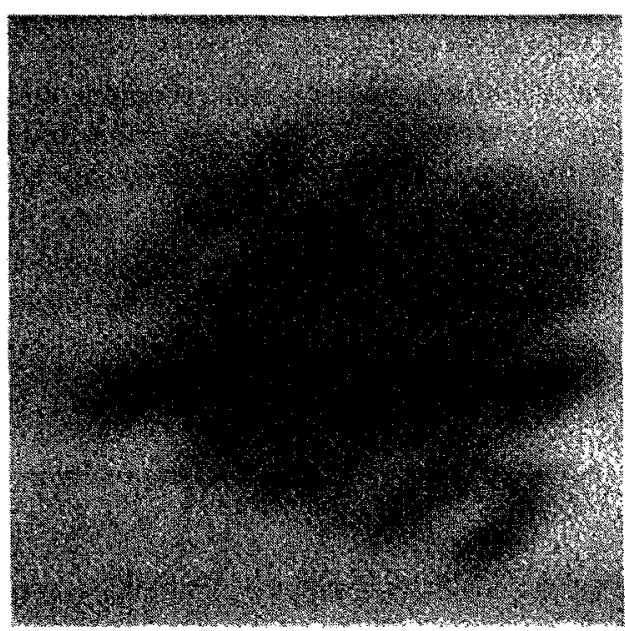

Fig. 4. Experimental DC-MFM image of perpendicularly magnetized bit in Co/Pt multilayer sample; scan $5 \times 5 \mu \mathrm{m}$.

\section{CONCLUSIONS}

We have shown that there is a direct equivalence between the MFM imaging process and the inductive readout process in magnetic recording. This has been demonstrated in both spatial and spatial frequency domains. For the specific case of perpendicularly magnetized media, MFM imaging via a simple rectangular 'monopole' tip generates an identical response, apart from constant factors, to inductive readout via a ring-type head assuming equal tip/gap widths and tip/head to sample separation. Thus (DC) MFM imaging offers no apriori benefits in terms of resolution to conventional readout systems used in magnetic recording, any resolution enhancements that are obtained arise as a result of scaling factors.

\section{REFERENCES}

[1] Kyusik Sin, P Glijer, J M Sivertsen and J H Judy, "Magnetic force microscopy studies of ultrahigh density magnetic recording on longitudinal and perpendicular media', IEEE Trans Magn, vol 33, pp 1052-1057, March 1997.

[2] P Glijer, E N Abarra, H Kisker and T Suzuki, MFM studies of recording phenomena in high density longitudinal recordings', IEEE Trans Magn, vol 32, pp 3557-3562, Sept 1996.

[3] P Rice and J R Hoinville, 'Spatial correlation between magnetic force microscope images and recording head output', IEEE Trans Magn, vol 32, pp 3563-3565, Sept 1996.

[4] C D Wright and E W Hill, 'Reciprocity in magnetic force microscopy', Appl Phys Lett, vol 67, pp 433-435, July 1995 .

[5] B K Middleton, C D Wright, S R Cumpson and J J Miles, 'Output waveforms in the replay process in digital magnetic recording', IEEE Trans Magn, vol 31, pp 2365$2379,1995$.

[6] C D Wright and E W Hill, 'Reciprocity based transfer function analysis in magnetic force microscopy', Appl Phys Lett, vol 68, pp 1726-1728, March 1996.

[7] C D Wright and E W Hill, 'A reciprocity-based approach to understanding magnetic force microscopy', IEEE Trans Magn, vol 32, pp 4144-4146, Sept 1996.

[8] H N Bertram, Theory of Magnetic Recording, Cambridge University Press, Cambridge, 1994.

[9] C D Mee and E D Daniel, Magnetic Recording Technology (2nd Ed), Chapter 2 (Eq. (2.29), B K Middleton, 'Recording and reproducing processes', McGraw Hill, 1990.

[10] Xiaodong Che et al, Noise analysis and image focusing for magnetic force microscopy', J Appl Phys, vol 73, pp 5805-5807, May 1993.

[11] C D Wright, private communication to $\mathrm{H} \mathrm{N}$ Bertram, August 1996.

[12] C D Wright, "Magnetic force microscopy using the Burleigh Personal AFM', Burleigh Applications, March 1996. 